Send your letters to the Editor, British

Dental Journal, 64 Wimpole Street, London W1G8YS E-mail bdj@bda.org

Priority will be given to letters less than 500 words long. Authors must sign the letter, which may be edited for reasons of space.

\section{Phossy jaws}

Sir, a five-year-old girl visited our clinic with an extraoral swelling located on the lower left side of her face that had been there for two days. On clinical examination, severe dental caries extending up to the pulp (many of the teeth with only root stumps left) was seen in all of the deciduous teeth except the lower anteriors. The patient had a history of chronic matchstick consumption for the past two-and-a-half years until it was noticed and stopped by her parents. The child had also drunk milk from a bottle for the last three years. Seeing the child's condition, 'nursing bottle caries' was the instant diagnosis. However, the matchstick consumption reminded me of an occupational hazard associated with matchstick (phosphorus) consumption which has long been eradicated: 'phossy jaws'.

'Phossy jaws' was osteonecrosis of the jaw caused by exposure to white phosphorus during the manufacture of matches. They were made by dipping the match ends into a mixture containing white phosphorus. ${ }^{1,2}$ Factory workers were exposed to fumes from the white phosphorus during mixing and spreading of the dipping material, and the drying and boxing of the matches. ${ }^{2,3}$

About 11\% of those exposed to phosphorus developed the disease. The average period from first exposure to diagnosis was five years. The mandible and maxilla could be affected. Phossy jaws were seen mostly in children, adolescents and women who worked in match factories up to 16 hours every day. Phosphoric vapour is generated by heating up phosphoric compounds, and is also absorbed through the gastro-intestinal tract. Dental decay was considered a prerequisite, leading to more serious problems like periostitis and osteomyelitis. Phossy jaw was fatal in about $20 \%$ of cases, usually because of septicaemia or meningitis. ${ }^{1,3}$

The discovery of red phosphorus led to successive prohibition of white phosphorus throughout the industry and after prohibition of the use of red matchsticks and matchstick making industries, this occupational disease was eradicated. ${ }^{4}$

In our case, no other finding was recorded apart from caries and facial swelling. Thus, the aim of this letter is to make readers aware of the 'matchstick menace' of the past.

\section{Kamboj}

Lucknow

1. Donoghue M A. Bisphosphonates and osteonecrosis: analogy to phossy jaw. Med J Aust 2005; 183: 163-164

2. Carter G, Goss A N, Doecke C. Bisphosphonates and avascular necrosis of the jaw: a possible association. Med J Aust 2005; 182: 413-415.

3. Purcell P M, Boyd I W. Bisphosphonates and osteonecrosis of the jaw. Med J Aust 2005; 182: 417-418.

4. Myers M L, McGlothlin J D. Matchmakers' 'phossy jaw' eradicated. Am Ind Hyg Assoc J 1996; 57: $330-332$.

\section{DOI: 10.1038/bdj.2007.1057}

\section{Peritonsillar abscess}

Sir, reading $B D J$ letters on Unexpected quinsy (BDJ 2007; 203: 227) reminded me of a recent event.

A 61-year-old male was referred from his GP to investigate a pain of ostensibly dental origin down the right side of the throat. The pain had started a week earlier, and made swallowing very painful. The GP has prescribed coamoxiclav, which had had no effect on the pain.

On examination, there was no swelling but it was clear that the patient was in moderate to severe discomfort. There was a reddened area around the soft palate, extending down the fauces, to the retromolar area of the right mandible. This appeared to extend down the lingual side of the mandible. There appeared to be no areas of suspicion from a dental point of view, however, I requested a minimal DPT view of the right sextant and advised the patient to continue with the co-amoxiclav in the meantime. I did advise him and his wife of the potential for throat involvement and to be wary of stridor.

The DPT was inconclusive, and nothing abnormal could be detected. On contacting the patient, I was told that he was comfortable and that the pain

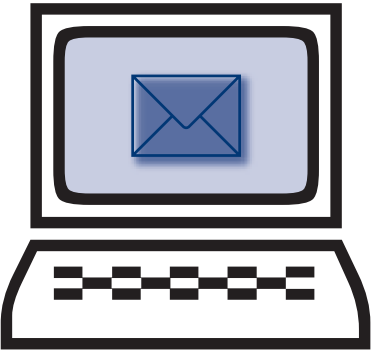

was lessening. I advised him to remain vigilant, and to return in seven days for review, or sooner should it recur.

Two days later, I was contacted by the on-call ActE doctor, requesting my opinion. The patient had turned up in severe pain from the same area, but the doctor was at a loss to explain it. No stridor, but the same area was now swollen as well as reddened.

I saw the patient, and noticed that the residual tonsillor tissue on the lateral border of the tongue was reddened and that the rest of the right side of the fauces was slightly swollen without pointing. It was soft and quite fluctuant to touch, rather than the firm swelling one may see with cellulitis. I decided to drain the area, but on incision gained no pus at all, merely normal bleeding.

Because of the fact that he was obviously in considerable distress and having problems with drinking fluids I referred him directly to Raigmore Hospital in Inverness, with the differential diagnosis of 'Quinsy'. This was confirmed by ENT surgeons, and successfully treated with IV antibiotics.

It is interesting to note that the ENT surgeons initially refused to believe that it was a dentist who had diagnosed the problem and kept asking the patient for the name of the referring 'doctor'.

This episode highlights the fact that 'quinsy', or peritonsillor abscess, is not just a potential problem of the young, with active tonsils, but can recur in the mature patient via the residual tonsillor tissue in the fauces and lingual regions. It can arise de novo and can present as a problem with moderate to severe pain, with swelling, fever, malaise and headache, along with hoarseness described as 'hot potato voice'. The jugulodigastric nodes may be involved, but this case was entirely localised to the area of infection. The diagnosis can be easily dismissed as a simple 'sort throat', but if the symptoms persist or recur, then peritonsillar abscess should be considered as a potential diagnosis.

\section{Monks}

Isle of Skye

DOI: 10.1038/bdj.2007.1058 


\section{Labiogingival notch}

Sir, the labiogingival notch is a developmental phenomenon that can be easily misdiagnosed and improperly treated. The labiogingival notch seen on the central incisors was first described by Brin and Ben-Bassat in 1989 in a population survey of 1,880 Israeli school children. They found a labiogingival notch in 123 children, with 6.5\% prevalence on at least one central incisor. In 96 (5.1\%) of the children, the notch appeared unilaterally, while in 27 children (1.4\%), the notch appeared bilaterally. ${ }^{1}$ Later in 2001 they reported two cases of labiogingival notch which were misdiagnosed as pathological condition and were about to undergo unnecessary invasive procedures.

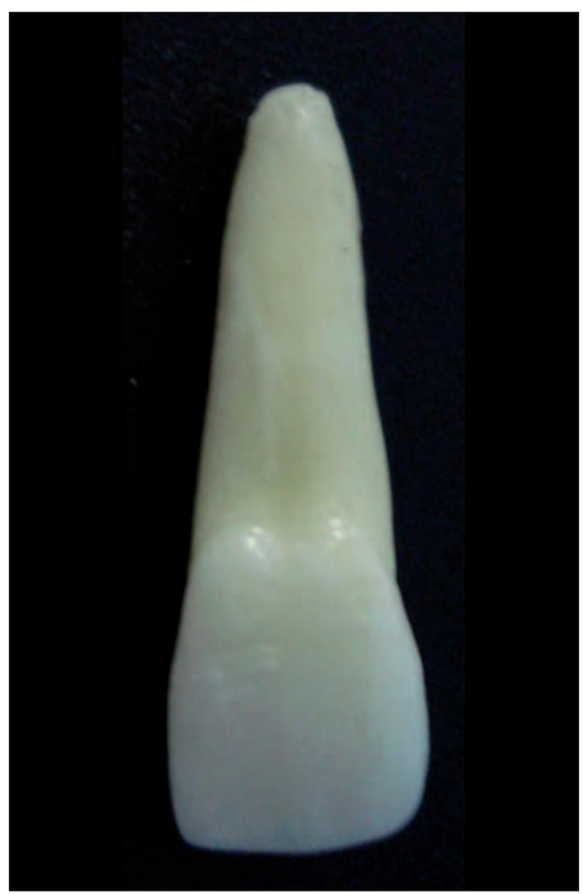

Fig. 1 Labiogingival notch on left upper centra incisor tooth, seen as a shallow depression near the CEJ

Corresponding to their description of labiogingival notch, I found a similar defect on the labial aspect of the upper central incisor while examining extracted teeth (Fig. 1). With this description of labiogingival notch, I would like to emphasise the importance of recognition of such a defect from cervical carious lesion treatment which may lead to an unnecessary invasive treatment. The labiogingival notch appears as an enamel depression close to the cementoenamel junction, with a depth that varies from a shallow depression to a deep groove. It can be identified by using a periodontal probe. The gingival margin closely follows the enamel contour, appearing almost normal in the case of a shallow notch, while in the case of a deep notch, it acquires an irregular contour because of extension of the gingival tissue into the defect. Many consider it a defect due to trauma during childhood. ${ }^{2}$ Brin and Ben-Bassat also noted this defect in children who suffered injury to their deciduous teeth. Thus one must enquire about the possibility of any injury during childhood when such a defect is noted. Clinically it may or may not pose a problem depending on the depth of the defect. A shallow defect may not be visible unless probed, while a deeper defect may require treatment for aesthetic purposes. In such cases placement of a restoration and gingival recontouring may be considered.

\section{Juneja}

Lucknow

\section{Ben-Bassat Y, Brin I. The labiogingival notch: an anatomical variation of clinical importance. J Am Dent Assoc 2001; 132: 919-921. \\ 2. Brin I, Ben-Bassat Y. Appearance of a labial notch in maxillary incisors: a population survey. $\mathrm{Am} \mathrm{J}$ Phys Anthropol 1989; 80: 25-29.}

\section{DOI: 10.1038/bdj.2007.1059}

\section{A distinctive entity}

\section{Sir, I would like to comment on the} $B D J$ editorial (BDJ 2007; 203: 289) written by Professor Jonathan Shepherd, who is also Vice Dean of the Faculty of Dental Surgery, Royal College of Surgeons (Eng). In the editorial, Professor Shepherd commented on the development of primary dental care, saying whether it has emerged as a distinctive entity characterised by a unique body of scientific knowledge applied in practice. Professor Shepherd also drew comparisons between primary dental care and primary medical care in academic foundations, saying the former was insecure.

I would like to point out that primary dental care is unequivocally a distinctive entity. Practitioners in primary dental care are able to undertake courses and programmes that are relevant to their practice and practise. Not only are these courses and programmes well researched and evidence-based with relevance to primary dental care, but they also provide opportunities for dental surgeons to share knowledge, understanding and most importantly, best practice. I do agree with Professor Shepherd that universities are an important source for research, but they are not the only source. Many practitioners in primary dental care would argue that university research is not applicable to their work when compared to other more invaluable sources that may be more pertinent to primary dental care.
I was confused as to why Professor Shepherd drew academic comparisons between primary dental care and primary medical care, which are both distinctive and separate entities. It was incorrect to compare the two, since each setting provides its own type of service in an environment to patients that have different needs. Primary dental care may be less academic than primary medical care, since the former is more practically oriented in its approach to patient care. Professor Shepherd professed to this by saying that the overlap between primary and secondary dental care is much less defined than it is in medicine.

Finally, the editorial by Professor Shepherd illustrated bias. As Vice Dean of Faculty of Dental Surgery, Royal College of Surgeons (Eng), Professor Shepherd took every opportunity to promote the importance of the Faculty to the role that is performed by primary dental care practitioners. He must understand there are many postgraduate education providers of varying sizes that provide better teaching and training, producing more relevant and more successful outcomes than the career pathways set out by the Faculty.

S. Shah

Epping

DOI: 10.1038/bdj.2007.1060

\section{Tilting at windmills}

Sir, Dr Yeung (BDJ 2007; 203: 291-293)

is mistaken in citing the WHO Report $916^{1}$ as evidence of a causal role for sugar in obesity, and consequently in those diseases associated with obesity. That report, in common with other recent expert committee reports, ${ }^{2,3}$ concluded that no such role is indicated by the evidence. Thus it is speculative to assert (BDJ 2007; 203: 122) that the measures proposed by the Faculty of Public Health 'Position Statement' intended (but in no case demonstrated) to reduce sugar consumption would reduce the prevalence of obesity, or materially influence those diseases associated with obesity.

He should also note that the review of evidence of effectiveness of oral health promotion methods by Professor Liz Kay for the Health Development Agency also failed to find evidence that attempts to alter sugar consumption were an effective means of reducing dental caries prevalence. ${ }^{4}$ In contrast, fluoride toothpaste use has been demonstrated to be highly effective but, unfortunately, is not yet universal, even in this country. ${ }^{5}$

Public health is best served by professional support of evidence-based and 
practical interventions, not by tilting at windmills, however fashionable.

\section{R. Cottrell}

\section{The Sugar Bureau}

1. World Health Organisation/Food and Agriculture Organization. Diet, Nutrition and the Prevention of Chronic Diseases. Report of the Joint WHO/FAO Expert Consultation.Technical Report Series no. 916. Geneva: World Health Organization \&t Food and Agriculture Organization, 2003.

2. Food and Nutrition Board, Institute of Medicine. Dietary reference intakes for energy, carbohydrates, fiber, fat, protein and amino acids (macronutrients). Washington: The National Academies Press, 2002.

3. Health Council of the Netherlands. Dietary Reference Intakes: energy, protein, fats and carbohydrates. The Hague: Health Council of the Netherlands, 2001.

4. Kay E J. Caries prevention: based on evidence? Or an act of faith? Br DentJ 1998: 185: 432-433.

5. Walker A, Gregory J, Bradnock G, Nunn J, White D. National Diet and Nutrition Survey: young people aged 4-18 years. Volume 2. Report of the Oral Health Survey. London: The Stationery Office, 2002.

DOI: 10.1038/bdj.2007.1061

\section{High quality, less bias}

Sir, we enjoyed the paper by Sathorn \&t Parashos $^{1}$ on evidence-based patient care. However, we were concerned that section 7.0 'Where can the evidence be found' missed some vital information. Specifically, section 7.1.5 on The Cochrane Library was strangely incomplete. The authors conclude that 'currently this database has little benefit, if any in the dental field'. We would like to challenge this opinion with the following information which might be helpful for the readers of the $B D J$ :

The Cochrane Library contains several databases and each are of considerable value to searching for evidence in dentistry. These include:

1. Cochrane Database of Systematic Reviews (CDSR). These are Cochrane reviews and recognised as the highest quality systematic reviews in biomedicine. In the latest issue (Issue 3 , 2007) there are 3,197 complete reviews and 1,744 protocols. Of these 74 reviews are in dentistry including: restorative dentistry, periodontology, implantology, orthodontics, paediatric dentistry, endodontology, oral surgery, oral medicine, oral pain, oral cancer, craniofacial anomalies and dental public health. Also included are 66 protocols for forthcoming systematic reviews in dentistry. CDSR should help to reduce duplication of effort listing existing and 'in progress' systematic reviews
2. Database of Abstracts of Reviews of Effects (DARE). This is a unique database from the University of York and includes critically appraised non-Cochrane reviews. A simple search of 'Dentistry' OR 'Oral Health' indicates at least 70 reviews currently. The critical appraisal is helpful in guiding the reader to the potential value of the reviews

3. Cochrane Central Register of Controlled Trials (CENTRAL). This register is the most complete database of trials and related publications as it includes the international handsearching programme from Cochrane volunteers. As a result, many trials have been found that were not originally included in other databases such as MEDLINE. In the current issue, there are more than half a million records.

Systematic reviews should be the starting point for any search for information since the hard work of finding, appraising and summarising the information has already been done. The quality of the review is crucial and several surveys have demonstrated that Cochrane Reviews are of higher quality and less biased than other systematic reviews. ${ }^{2,3}$ Readers looking for evidence in dentistry will find much of relevance to their practice in The Cochrane Library whether as Cochrane reviews or in the DARE database. If you are looking for controlled clinical trials then again CENTRAL is the most complete database including oral health. The Cochrane Collaboration and the Oral Health Group recognise that there is still a huge amount to do in producing best evidence but we also hope that the results so far will help to inform and improve dental practice and policy.

I. Needleman, H. Worthington,

L. Fernandez-Gonzalez, P. Brunton, J. Clarkson, P. Coulthard, M. Esposito, A-M. Glenny, L. Hooper, V. Marinho,

D. Moles, W. Shaw on behalf of Cochrane Oral Health Group Editorial Team

1. Sathorn C, Parashos P. Questions and answers in evidence-based patient care. Br Dent J 2007; 203: 309-319.

2. Moher D, Tetzlaff J, Tricco A C, Sampson M, Altman $D$ G. Epidemiology and reporting characteristics of systematic reviews. PLoS Med 2007; 4: e78.

3. Glenny A M, Esposito M, Coulthard P, Worthington $\mathrm{H} \mathrm{V}$. The assessment of systematic reviews in dentistry. Eur J Oral Sci 2003; 111: 85-92.

DOI: 10.1038/bdj.2007.1062 